\title{
USE OF MOBILE APPLICATIONS IN SECURITY AND SAFETY SYSTEMS
}

\author{
Andriy VOLKOVIY and Ilya TIKHOV
}

\begin{abstract}
This paper presents an analysis of the possibilities of using the mobile devices (especially smartphones) with specialized software as parts of distributed systems that perform security and/or safety functions. It provides an overview of mobile-based security and safety applications. Finally, it outlines a generalized model of security and safety systems based on Web-services or systems of mobile phone network operators.
\end{abstract}

Keywords: Mobile phone, smartphone, GPS, Web-service, XML, RSS, early warning system, alarm receiving system.

\section{Introduction}

Mobile phone with computer features is no longer an exception. Rather it is hard to find among modern products a mobile phone that is only cellular telephone without additional options like abilities to interact with computerized systems (PC, laptops, etc.) and to access the Internet (Web, e-mail, etc.). Nowadays mobile technologies are rapidly developing. There are lots of people who are ready to refuse desktop computers and other stationary communication devices and go to mobile (portable) devices. As the performance and functionality of mobile devises grow, the number of people who wants to stay in touch using just small device that can fit in their pocket is rapidly increases. It's essential to note that performance of mobile devices is not really inferior to desktop PC, and problems of human-machine interface is quite successfully solving with touch screen technologies and mobile operation systems. It's also important that modern mobile applications don't use performance of mobile devices in full. At the same time the presence of additional interfaces (Bluetooth, Wi-Fi, etc.), supplementary services (PTT, instant messaging, etc.) and extra build-in modules (GPS receivers, video cameras, etc.) form good background for different solutions based on mobile platforms in various application areas.

For example, ordinary GSM mobile phone with middle-resolution camera and GPRS/EDGE connection is able to execute client software that read and recognize 
bar code for transmission data about merchandise in storage to server software (database management system).

Objectives of the paper are to determine possibilities of using modern mobile devices for security and safety solutions and to represent generalized models of such applications.

\section{Trends in mobile technologies}

One of the most important steps in of mobile phones development is embedding functionality of modern PC into mobile phone. In result we've got portable computer that had its own operation system to launch different applications and ability for calling. These devices were called smartphones. They are widespread all over the world and are the most popular between all mobile devices.

Modern models of mobile phones typically also serve as electronic organizers, portable media players and photo/video cameras (camera phones) with high-resolution touchscreen, GPS navigation, Wi-Fi and mobile broadband access.

A smartphone runs a complete mobile operating system. Widespread examples are Apple iOS, Google Android, Microsoft Windows Phone 7, Nokia Symbian, Research In Motion BlackBerry OS, and embedded Linux distributions such as Maemo. Such systems can be installed on many different models of mobile devices. They can run third-party applications, using an application programming interface (API).

According to Olswang report in early 2011, the rate of smartphone adoption is accelerating: as of March 2011 22\% of UK consumers had a smartphone, with this percentage rising to $31 \%$ amongst $24-35$ year olds. ${ }^{1}$

Growth in demand for advanced mobile devices boasting powerful processors and graphics processing units, abundant memory (FLASH memory), high-resolution screens with multi-touch capability, and open operating systems has outpaced the rest of the mobile phone market for several years. ${ }^{2}$ According to an early 2010 study by ComScore, over 45.5 million people in the United States owned smartphones out of 234 million total subscribers. ${ }^{3}$ In March 2011 Berg Insight reported data that showed global smartphone shipments increased by 74 percent from 2009 to $2010 .{ }^{4}$ Available information show that the number of smartphones, and therefore the number of smartphone users grows.

\section{Smartphones, services and web-services}

As smartphones have access to the Internet and can launch different applications these mobile applications are able to use defined web-services for getting data from outside. A Web-service is a software system designed to support interoperable ma- 
chine-to-machine interaction over a network. It has an interface described in a machine-processable format (specifically WSDL). Other systems interact with the Webservice in a manner prescribed by its description using SOAP messages, typically conveyed using HTTP with an XML serialization in conjunction with other Web-related standards.

A Web-service is an abstract notion that must be implemented by a specific agent. The agent is the special piece of software or hardware that sends and receives messages, while the service is the resource characterized by the abstract set of functionality that is provided. To illustrate this distinction, you might implement a particular Web-service using one agent one day (perhaps written with one of programming languages), and a different agent the next day (written with another programming language) with the same functionality. Although the agent may have changed, the Webservice remains the same.

The purpose of a Web-service is to provide some functionality on behalf of its owner - a person or organization, such as a business or an individual. The provider entity is the person or organization that provides an appropriate agent to implement a particular service. A requester entity is a person or organization that wishes to make use of a provider entity's Web service. It will use a requester agent to exchange messages with the provider entity's provider agent. In order for this message exchange to be successful, the requester entity and the provider entity must first agree on both the semantics and the mechanics of the message exchange. ${ }^{5}$

A striking example of Web-services use is applications that display weather conditions and weather forecast at the screen of smartfone. Additional features that allow determining the location (by GPS, mobile devise localization in network through TDOA or E-OTD techniques, etc.) make other services very popular. According to a ComScore report released on May 12, 2011, nearly one in five smartphone users are tapping into check-in services like Foursquare and Gowalla. A total of 16.7 million mobile-phone subscribers used location-based services on their phones in March $2011 .^{6}$

There are a lot of applications created and submitted to application's store, and most of them are targeted on entertainment and multimedia. But there are part of applications that really may be useful in people life, may affect behavior and feature of people. Such applications were created with mission to help and to save people life at any cost, respond on any changes and give sufficient estimate of situation, help to make right decision as fast as possible. 


\section{Mobile applications for personal safety}

There are a lot of natural cataclysms and human-caused events that take lives, harm the environment, and cause huge financial damage (e.g. floods, forest fires and other natural disasters, etc). Existing early warning systems are designed to coordinate preventive and rescue actions under the guidance of the relevant authorities and rescue services (usually governmental agencies). ${ }^{7}$ Purpose of such actions is to reduce potential losses and first of all the number of victims among the population. Nevertheless, natural disasters continue to take lives, and among the reasons are both unexpected appearance of threats and untimely getting news about these dangers. And if to know in advance about disaster coming is not always possible, contrariwise raising possibilities of information systems can and should be constantly done.

Below are examples of applications that are ready to try to help in a danger situations. If we look for "life-saving" applications in iTunes for iPhone mobile phone we can find:

- $\quad$ RESCUE - Endorsed by paramedics, fire fighters, Search \& Rescue groups and police officers. This is the smartest investment in your safety, you will make. It is a Personal Locator Beacon and Protection System all in one;

- $\quad$ iMapWeather Radio - With iMapWeather Radio you will receive critical voice and text alerts on life-threatening weather events. Your iPhone will "wake up" and also "follow you” with alerts wherever you go. Listen with ease to local weather forecasts while you are on the move. Enjoy the power of a Weather Radio, with all the convenience and precision of a smart phone.

The marketplace for Android applications suggests us such variants:

- Earthquake alert! - offering latest Magnitude 1.0 and higher earthquakes from all over the World;

- Real Time Warning alerts users of natural disasters on a local and global scale. It allows views on earthquakes, cyclones, volcanic eruptions, hurricanes, floods on an interactive map, as well as real time warnings of natural disasters; setting custom disaster alarms; setting monitors for earthquakes, cyclones, volcanic eruptions, hurricanes, floods; sharing option with Facebook Connect; and GPS functionality.

On the application market for one of the freshest mobile operation systems Windows Phone 7 we found 'TSUNAMI' that provides simple tutorials and illustrations, covering the following themes: Tsunami introduction, Earthquake introduction, Physics of waves, Physics of tsunami, Tsunami cycle, Wave propagation, Tsunami warning system, Measuring earthquakes, and Tsunami vocabulary. 
One of the applications developed especially for mobile phones on OS Windows Phone 7 is Touch-and-Save. ${ }^{8}$ This application provides an ability to get alerts about approaching natural disasters and other useful features for preserving user's safety. For the users, Touch-and-Save provides the following functions:

- gathering actual information about earthquakes, floods and eruptions from information services;

- visual displaying disaster situations in the world (the world map with zooming is used) and description for any incident (marker at the map) on request;

- determine the current location via GPS and displaying the current location at the map;

- notifications about appearance of new event or approaching dangers;

- customization of appearance, refreshment and notification options.

These functions provide an opportunity to obtain information necessary for making decisions about the reaction to the occurrence of dangerous natural disasters. The source of information is existing information web-services, such as:

- Worldwide Holocene Volcano and Eruption Information Service (available at the Global Volcanism Program site http://www.volcano.si.edu/);

- Global Disaster Alert and Coordination System (available at the Global Disaster Alert and Coordination System site http://www.gdacs.org/);

- Earthquake Data Portal (available at http://www.seismicportal.eu/).

Unfortunately there is no universal format for data on natural disasters and all of these systems provide information in different formats based on RSS and XML (e.g. Earthquake Data Portal uses QuakeML). As a result, use of each source of information requires not only web-address but also details of data representation.

Figure 1 shows the software system flowchart. Software is an application for Windows Phone 7 called Touch-and-Save. In "heart" of this application exists scheduler for usual refresh data, mechanism for analyze disasters' locations and compare with user's location. Appearance of the application is shown in Figure 2.

It's only one example among "defender" applications that is not perfect, but makes successful tries to help people, to warn people in time about approaching dangerous natural events and last decisions depend only on each person. Each of us have to decide what is the most important in his live and how to respond if ever the phone make a signal about approaching disaster. 


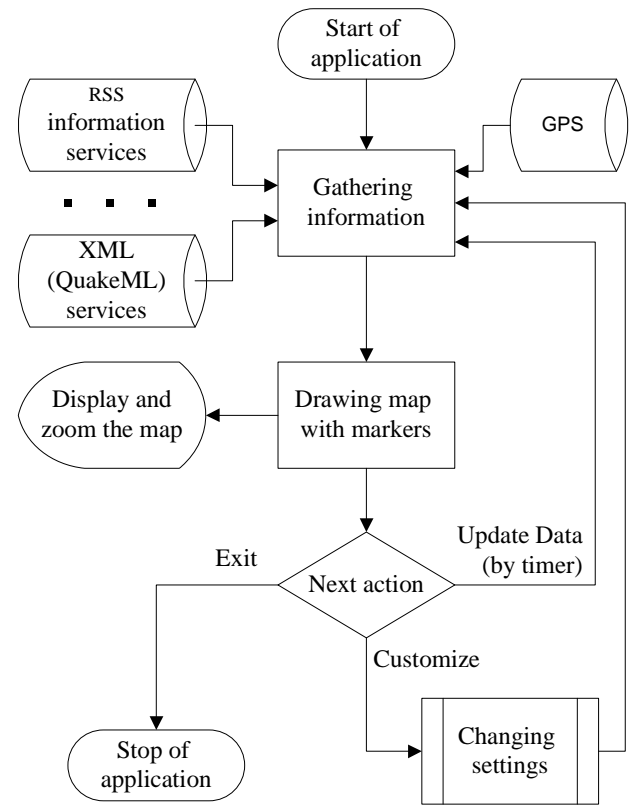

Figure 1: The system flowchart

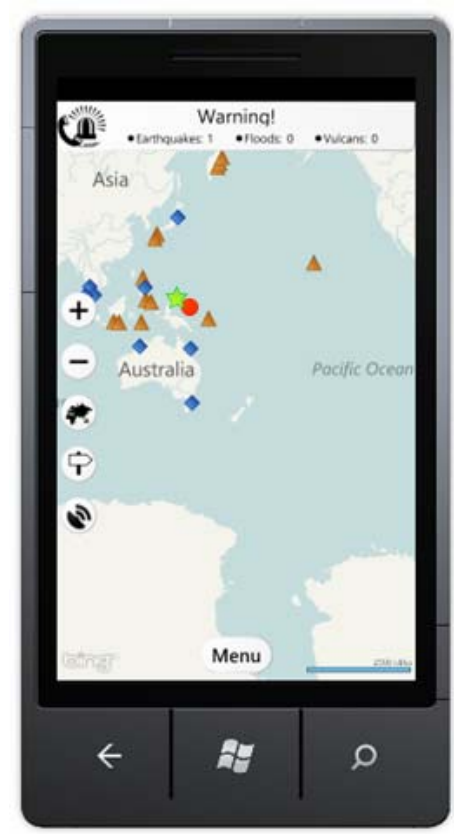

Figure 2: The application running in the mobile device emulator

\section{Mobile applications in the societal security systems}

Mobile applications for smartphones are still very specific solutions and do not solve the problems of security and safety in the complex. All the above examples are focused on obtaining information from the network and provide it to the user, i.e. in fact "defender" applications use one-way communication. Nevertheless, the potential of available solutions allows to speak about possibility of establishing a global security system, which includes smartphone as complete unit for human identification and condition (like lone worker device) that interacts not only with Web- and Mobile network services but also with alarm receiving centres. Figure 3 shows the model of societal security system where mobile devices are used as customers' terminals with two-way communication.

Feedback (communication from device to system) allows providing rescue service (through alarm receiving centre) with information necessary for search and rescue victim. Mobile applications can be component applications in which the functions are linked to services and all services use similar operation logic and open XML-based protocols. 


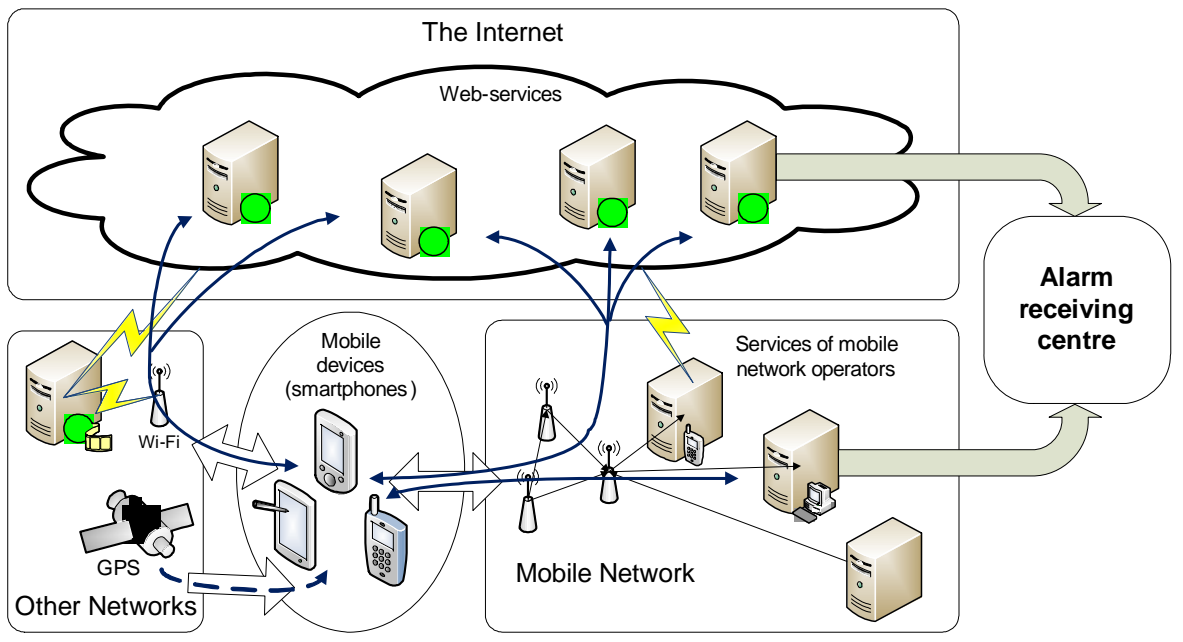

Figure 3: Architecture of the distributed societal security system.

\section{Conclusions}

Smartphones have the necessary resources to be used as part of systems able to implement security and safety features. The universality of such devices opens up broad prospects in terms of number of users. There are already some solutions that can solve certain problems of individual safety. At the same time there is no yet overall strategy to build systems for safety and security with mobile devices. For a comprehensive and effective solution of problems, associated with the use of mobile devices in societal safety systems, common approaches and methods defined by international standards and specifications are needed.

\section{Notes:}

1 Matthew Phillips and John Enser, "Does it add up? Olswang Convergence Survey 2011,” Olswang LLP, <www.olswang.com/convergence2011>.

2 "Smart phones: how to stay clever in downturn," 2009 TMT Predictions, $<$ www.deloitte.com/tmtpredictions $>$.

3 Antone Gonsalves, “Android Phones Steal Market Share,” InformationWeek, 7 April 2010, $<$ www.informationweek.com/news/224201881>.

4 Zach Epstein, "Berg: Smartphone shipments grew 74\% in 2010," Boy Genius Report (March 2011), <www.bgr.com/2011/03/10/berg-smartphone-shipments-grew-74-in-2010>. 
5 David Booth, Hugo Haas, Francis McCabe, Eric Newcomer, Michael Champion, Chris Ferris, and David Orchard, "Web Services Architecture," W3C Working Group (February 2004), <www.w3.org/TR/ws-arch>.

6 Lance Whitney, "Nearly 1 in 5 smartphone owners use check-in services," CNET News (May 2011), <http://news.cnet.com/8301-1023_3-20062640-93.html>.

7 Matthew L. Collins and Naim Kapucu, "Early Warning Systems and Disaster Preparedness and Response in Local Government,” Disaster Prevention and Management 17:5 (2008): 587-600.

8 Andriy Volkoviy, Ilya Tikhov, et al., "Web-Services-Based Mobile Application for Personal Safety,” Proceedings of CrISS-DESSERT 2011 (May 2011): 390-392.

ANDRIY VOLKOVIY, PhD, Associated Professor of Computer Systems and Networks Department of National Aerospace University named after N.E. Zhukovsky "KhAI.” Graduated from National Aerospace University as an engineer in Special-purpose computer systems in 2000. In 2006 he received a PhD degree in Automated control systems and advanced information technologies. He has published more than 30 articles dealing with problems of software engineering and assessment. E-mail: a.volkovoy@csac.khai.edu.

ILYA TIKHOV is a student of National Aerospace University named after N.E. Zhukovsky “KhAI." He also works part-time in DataArt IT company. His present research interests include methods of software development, particularly solutions for web and mobile devises. He interests in new technologies connected with the development of heavy interactive online solutions.E-mail: ilya.tikhov@gmail.com. 